\title{
DOE/R4/10642-TI \\ THE INSTALLATION AND OPERATION OF A PRODUCTION \\ SCALE MEMBRANE FILTRATION SYSTEM TO \\ TREAT AND REUSE WASTE WATER FROM A DYEHOUSE
}

\author{
DONALD A. BROWN \\ SENIOR FACILITIES ENGINEER \\ SARA LEE KNIT PRODUCTS
}

\begin{abstract}
:
The waste water from the dyeing of cotton and poly-cotton blends is characterized by high levels of salts and color. The discharged salts are felt to contribute to effluent toxicity and the color is aesthetically unacceptable in the receiving streams. The system of selective membrane filtration offers a method to address both of these problems. By treating only the waste water having the most color and salt concentration, the expensive membrane equipment can be considerably downsized. The volume of the color to be removed is drastically reduced as filtrate material. At these low volumes of color more economical means of removal can be employed. The membranes are designed so that the color and other pollutant materials are rejected but the salt solution is allowed to "pass through" the membrane. This produces clean water containing the recovered salt suitable for reuse, greatly reducing the salt content of the effluent. After the successful completion of a small pilot scale system, the installation and operation of a production sized system was desired prior to the full incorporation of this technology.
\end{abstract}

\section{KEY WORDS:}

Split drain, Ultra-filter, Nano-filter, brine, pressure jet, filtrate, reject, permeate, jet "drops", Programmed Logic Controller (PLC), and Physical/Chemical.

\section{SCOPE:}

The dyeing method most commonly used for knitted cotton fabric is to utilized programmed pressure jets. These typically add preset volumes of water, chemicals, and prepared dyes; control the dyeing times and temperatures; and remove the spent dyes and chemicals through a program of volume drops, fills, and rinses. The majority of the salt and color was previously proven to be contained in the first two "drops" from the jet. The small amount of color and salt removed in the subsequent rinses was deemed to have a minimal effect on the overall effluent (note chart 1). It was the scope of this project to evaluate on a large scale basis the feasibility of installing and operating a production scale system to treat and reuse the discharges from an actual production dye house. Previous smaller scale operations

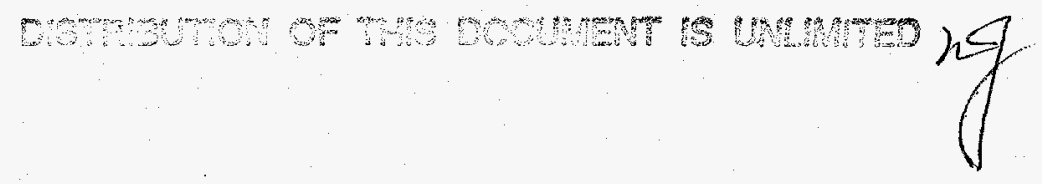




\section{DISCLAMIER}

Portions of this document may be illegible in electronic image products. Images are produced from the best available original document. 


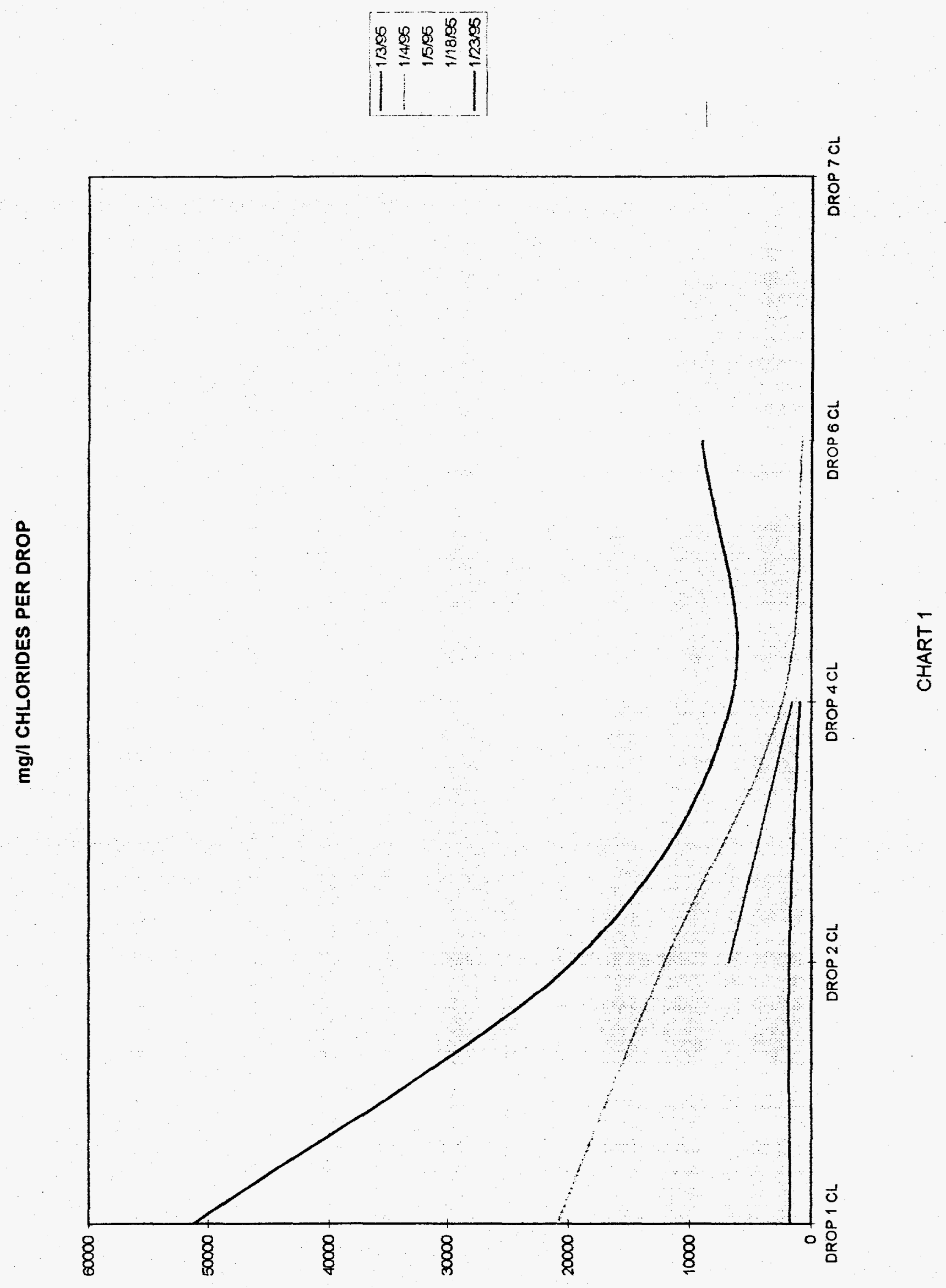



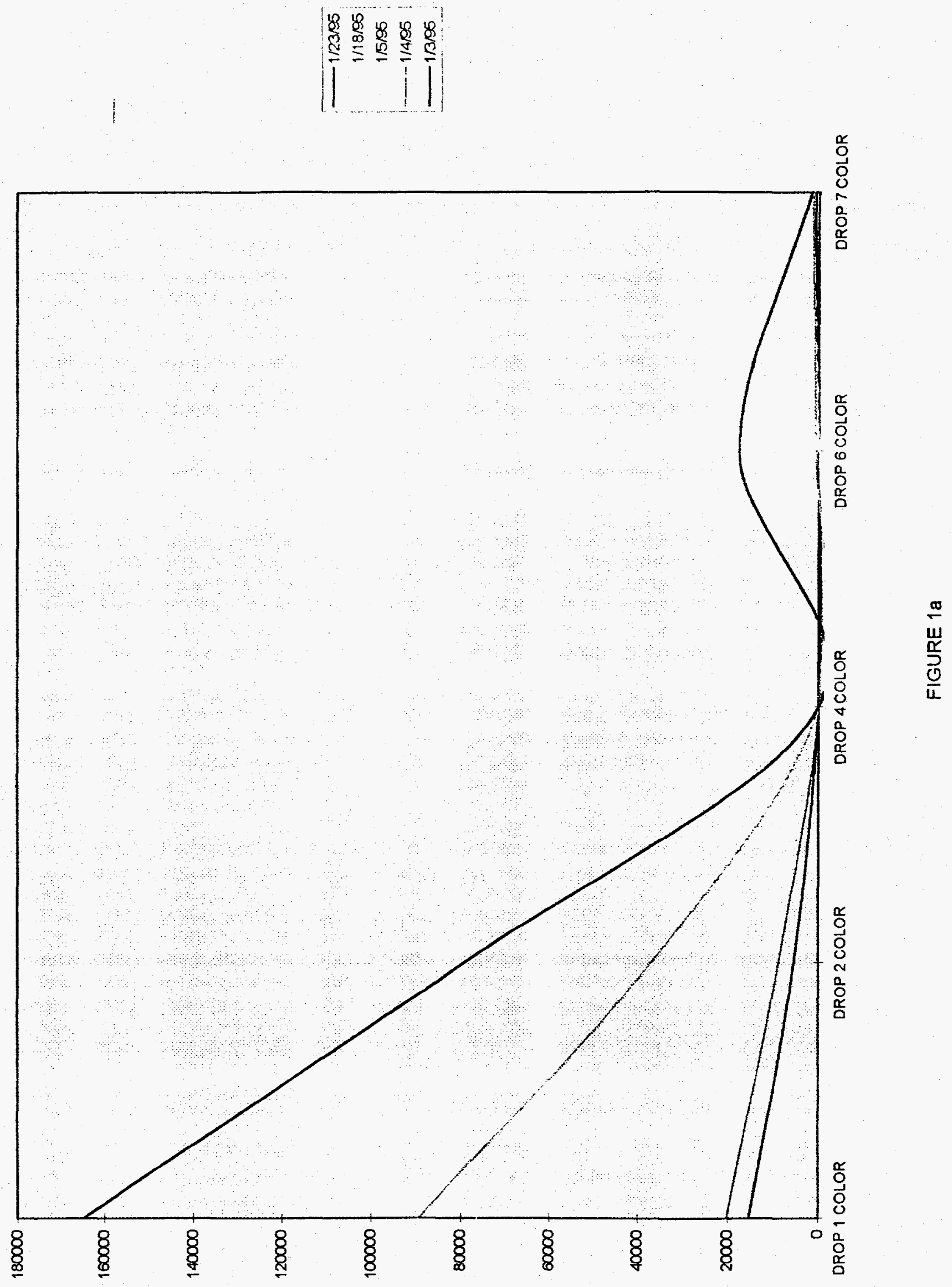
yielded such small volumes of reject material (the filtered dyes and other solid pollutants) that meaningful analysis of its treatment was difficult. There was an indication that the treatment would present difficulties both technically and economically. Prior to full incorporation of this technology, an analysis of this associated process was required. There is a concern that the increased cost of treatment of the concentrated filtered materials would offset any savings realized from the salt and water reuse.

Additionally to be fully incorporated were full size production dyeings with the reuse water. Controlled laboratory or sample dyeings fall short in defining the suitability and mechanics of using this treated water in full size equipment.

In order to resolve these questions a project was established to install and operate a membrane system capable of treating a volume of dyeing waste sufficient for reuse in production scale dyeings. Also a goal was established to install a Physical/Chemical system to evaluate and define methods and chemistries to treat the rejected filtrate materials. Another concern, and past failures of membrane systems, was the problem associated with the life and cleaning cycles of the membranes. The only means to address this concern is to operate the system continuously over a series of conditions.

To aid in the funding of this project a grant from the Department of Energy was sought and awarded.

\section{INSTALLATION:}

The first step in installing this system was to isolated the dye drops to the membrane system. As previously stated only those wastes having the most color and salts were to be treated. Furthermore, there are dyeings which utilize sodium sulfate instead of sodium chloride. The methodology of the system is to pass the sodium chloride and reject the dyes and other pollutants. Sodium sulfate will not pass the membrane and has the negative potential of crystallizing on the membranes as it is concentrated with each pass through the system. These crystals will damage the sensitive nano-filter membrane. Great care and time was given to program each dye jet and each color formula to direct only the first two drops of those dyeings with sodium chloride to our system. A collection/ lift station for the specialized waste was created by partially plugging the discharge line of this special drain line ( The storage tank is in effect the special trench into which the waste is dropped). A sump pump deliveries upon demand to the ultra-filter processing tank. The output from the ultra filter is delivered to the nano-filter processing tank ( note system layout in figure 2). The processed water from the nano-filter is discharged into a 250 gallon lift station where it is pumped to a 4000 gallon storage tank located on the roof of the dye house for subsequent re-use. Also located on the roof is a brine storage tank which deliveries a programmed metered flow of brine to the dyeing jets. For our installation we chose to utilize the existing metered line and valve at each jet selected for re-use. A simple programming change is enacted to meter the volume of reuse water containing salt to the jets. More specifically, in normal operations the PLC's will delivery the quantity of brine to the jets (several hundred gallons) to yield the correct concentration of sodium chloride when the jet is filled to its desired 


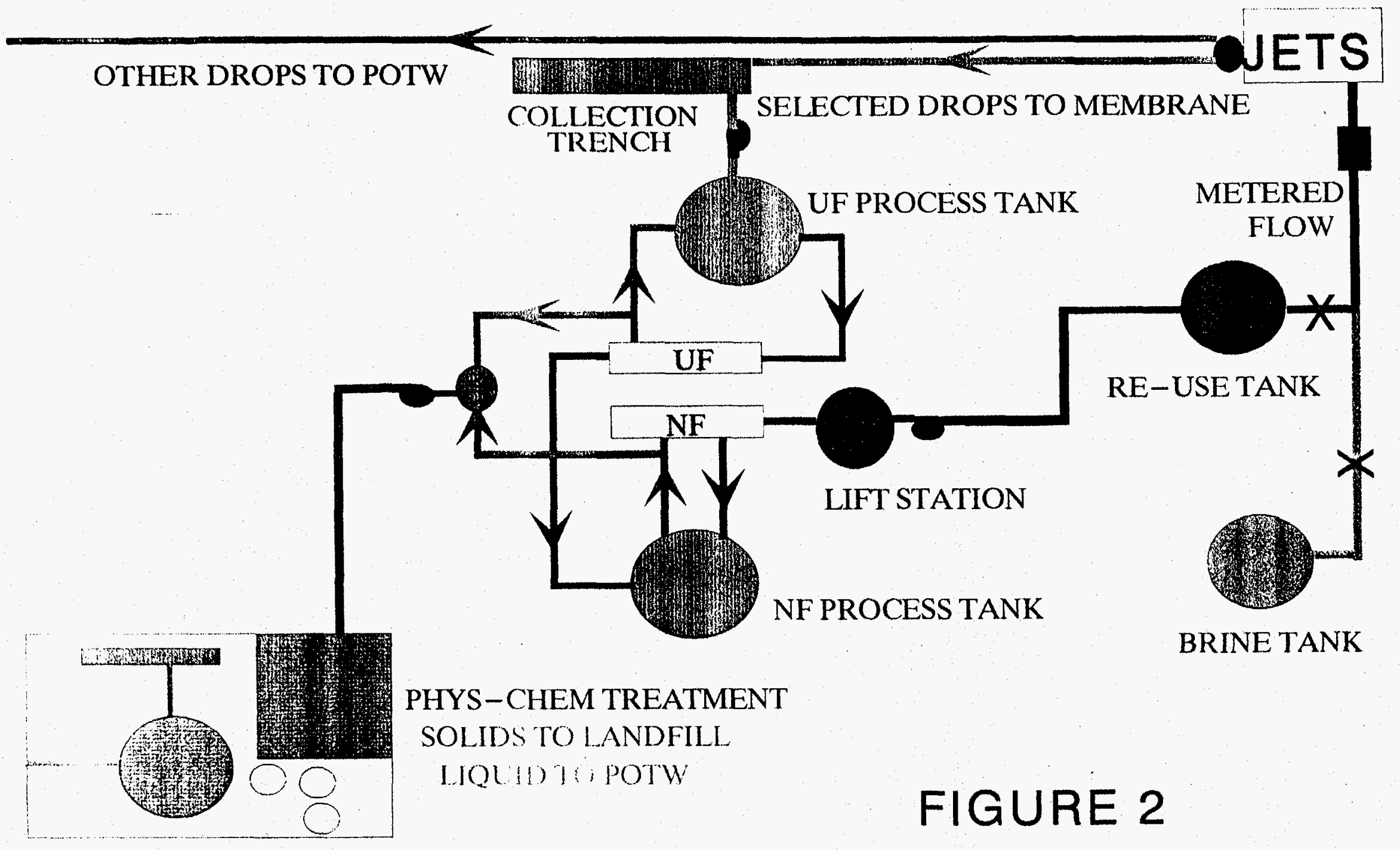


liquor volume. For the re-use scenario the solution in the storage tank is used to fill the volume of the jet ( 1500 to 2200 gallons). The volume of this solution is purposely metered just short of the correct jet volume so that the jet water fill "step" will activate and delivery the correct water volume. After a period of circulating with the fabric the specific gravity of the liquor is measured and if required additional salt is added

The resulting reject material from the filters can be directed to a reject storage tank as the membranes operate. During operation we have found however that it is better to selective pump the bottoms of the respective membrane process tanks. These tanks have conical bottoms and offer additional solid removal by settleability prior to being fed to the membranes (The feed to each membrane system from their respective process tank is located on the side of the tank at a point approximately $25 \%$ from the bottom). This concentrating of solids is especially noted in the ultra-filter process tank which contains a considerable amount of fabric lint. A physical /chemical treatment system is located adjacent to the membrane system to treat the accumulated reject waste.

\section{MEMBRANE OPERATION:}

Similar applications of membranes have resulted in the fouling and poor or no performance of the membranes. Prior to committal to a larger system it is necessary to estimate the life of the membranes and their maintenance in this application. This application is somewhat novel in that it incorporates two different membranes staged in series. The first stage ultra filter removes the larger particles which in past applications have "blinded" the finer and more selective second stage nano-filter. The ultra filter has continuously operated on one shift since late April and has produced acceptable flows (note chart 2). During periods of decreased flows an acid cleaning procedure has been established which returns the flows back to their specified level. (The implementation of the cleaning procedure is also evident on chart 2). Operation of the nano-filter is more limited (note system log data in appendix) but surprisingly much more successful than anticipated. The system was designed to process 7.5 gallons per minute. It will produce well in excess of 40 gallons per minute. It is theorized that these high efficiencies are the result of the lessened loading on the nano-filter created by the ultra-filter. The major constituent removed in the nano-filter is basically the reactive dyestuffs.

\section{PHYSICAL/CHEMICAL OPERATION:}

A concern with the system to be proven in this limited production scale operation was the ability to adequately treat the color and solids concentrated and removed by the membrane system. The previously mentioned small scale trials did not produce sufficient membrane reject material to adequately conduct tests. This system has provided enough reject material not only to continuously run and monitor a small scale Physical/Chemical treatment system but also evaluate additional and more economic chemistries. The present operation utilizes ferrous sulfate and a cationic polymer to precipitate the solids and color. A small amount of anionic polymer is later added to enhance floc 


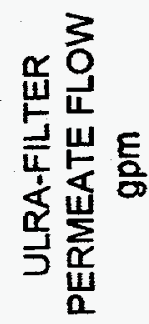

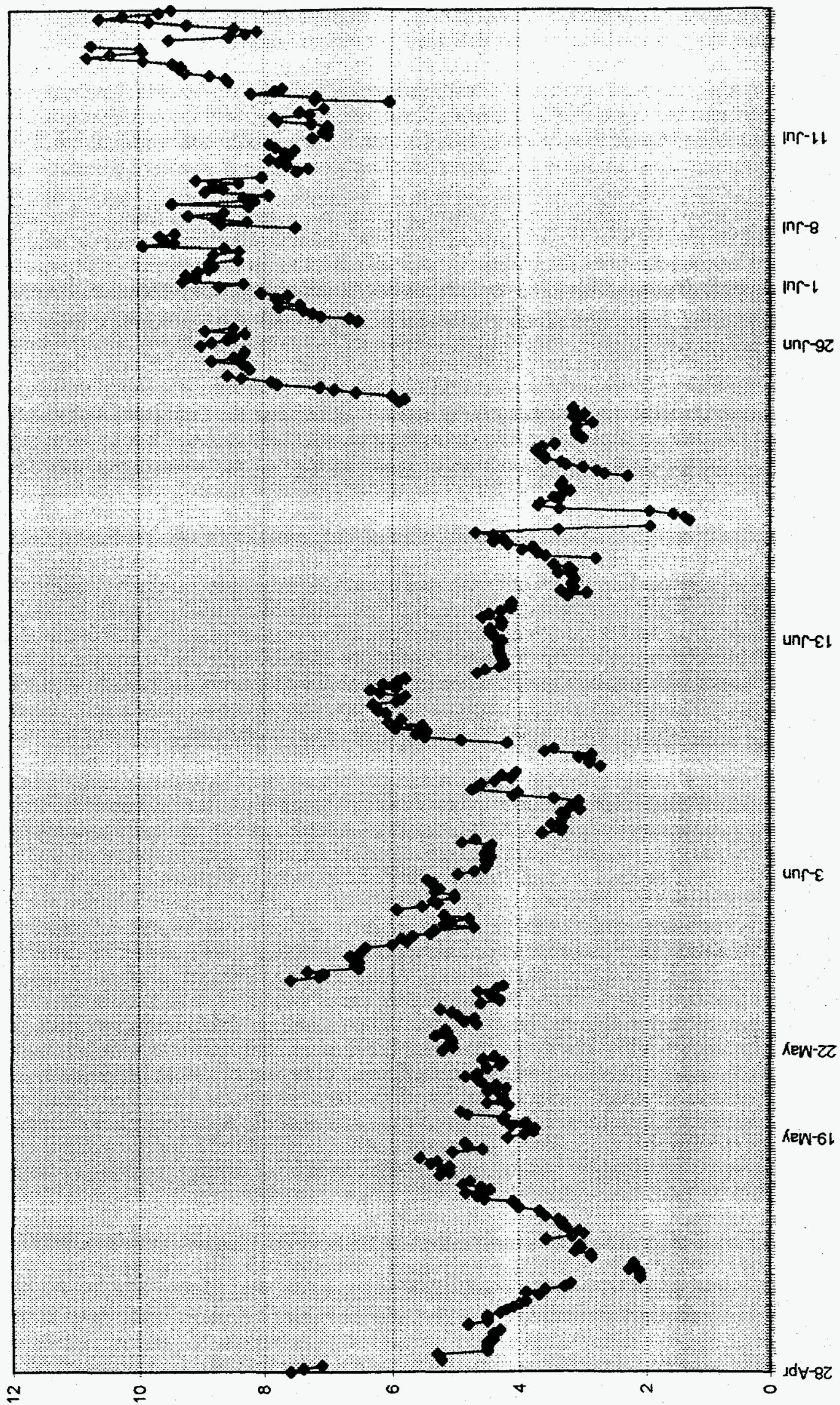

$\underset{\frac{N}{\alpha}}{\frac{1}{x}}$ 
settleability. This system has operated better that expected at an operating cost of about $\$ 2.00$ per 1000 gallons. It is producing effluent of good quality with only a trace of color.

\section{REUSE TRIALS:}

To date four full scale re-use trials have been conducted. Three were successful with no required color adjustments or procedural changes except for the aforementioned salt water loading changes. One dyeing was unlevel and required re-processing. It is felt that the cause of this defect can be attributed to difficulties in the dyeing of this particular shade or most probably the failure to adequately adjust the ph level of the re-use water prior to the addition of dyes. The influent to the membrane system is characterized by ph levels of 9.5 to 10.5 created by organic alkali's utilized in the dyeing process. $95 \%$ of this material is rejected in the filtration process and imparts a "non-buffered" high ph to the permeate. Only a very small amount of acid $(0.01 \%$ by volume $)$ is required to neutralize the water prior to re-use. This problem color has been repeated with acceptable results. All trial (re-use) dyeings have passed the quality fastness tests.

\section{SYSTEM IMPROVEMENTS:}

Additional modifications are planned to be made to the membrane system. One of these is to install a ph monitoring and adjustment system at the nano-filter effluent lift station. During the re-use trials some difficulties were encountered in attaining the correct (neutral ph) by adjusting directly into the jet. Excessive time is required for the higher ph in the fabric to be neutralized by the acid addition into the jet's chemical add system. Another item to be corrected that will facilitate the re-use is the installation of a booster pump on the feed line from the storage tank to the jets. The present gravity feed system adds approximately 15 minutes to the dyeing cycle.

The lint has created more frequent cleaning of the strainer basket prior to the ultra-filter than was anticipated. It is the intent to install a parallel filter so cleaning will not interfere with operation time. The lint is being controlled so that it does no adversely impact the ultra-filter. However, long term better lint removal would be of a major benefit to this project.

\section{ECONOMIC ANALYSIS:}

The economics of this system is based upon a more efficient removal of color and the re-use of the water and salt associated with the dyeings. During the installation of this project a significant improvement has been made in the dyeing process. The introduction and now increasing use of "low salt" dyes has lessened the salt concentration in the dye bath. In addition this new class of dyes has a 
much better exhaustion percentage than those dyes previously used. As more of these dyes are used the salt recovered and subsequent pay back on membrane system such as this is reduced.

However, the better exhaustion of these dyes offer additional significant economic advantages. Prior to the development and introduction of the "low salt" dyes our membrane design and economic analysis was based upon the need to capture two drop volumes of each dye jet in order to remove the color. This additional drop from the addition of clear rinsing water to the jets diluted the salt concentration collected for the membrane system to one half of that originally used. A problem was also created in that the system produced an excess of $100 \%$ of clean "salty water"(from two water volumes from each jet). A proposed solution for this excess water was to utilize reverse osmosis filters to concentrate the salt from the water and then reuse both constituents. All previous economic analyses on these systems were based upon the recovery of $50 \%$ of the salt and water. The enclosed analysis (note table 1) is reflective of the decreased salt concentration from the "low salt" dyes, the return of a greater percentage of the salt from those dyeings, the downsizing of the required capital with the decrease of volume to be treated, and the increase in treatment efficiencies as evidenced in this production pilot. This analysis indicates that an excellent payback can be realized from this equipment.

\title{
SUMMARY:
}

This project has proven that it is feasible to install and operate a membrane on a production scale basis to economically reduce the toxicity and color in receiving streams or the associated POTW's discharge. Every potential installation of these systems will require evaluations based upon regulatory issues, product mix, production curtailments, and current or future treatment costs. This system has operated beyond expectations. Evaluations will be continued to further define its operation in order to expand this system to all economically or regulatory applicable plants.

\section{DISCLAIMER}

\begin{abstract}
This report was prepared as an account of work sponsored by an agency of the United States Government. Neither the United States Government nor any agency thereof, nor any of their employees, makes any warranty, express or implied, or assumes any legal liability or responsibility for the accuracy, completeness, or usefulness of any information, apparatus, product, or process disclosed, or represents that its use would not infringe privately owned rights. Reference herein to any specific commercial product, process, or service by trade name, trademark, manufacturer, or otherwise does not necessarily constitute or imply its endorsement, recommendation, or favoring by the United States Government or any agency thereof. The views and opinions of authors expressed herein do not necessarily state or reflect those of the United States Government or any agency thereof.
\end{abstract}




\section{REPRESENTATIVE PLANT \\ COST EVALUATION \\ WITH LOW SALT DYE SCENARIO}

BASIS:

1 1ST AND 2ND DROPS EQUALS .12 MGD

2. ESTIMATE SALT COnc. TO BE $20.0 \mathrm{~g} / \mathrm{l}-\ldots-\ldots--$ WITH LOW SALT DYES

3. DYE FILTRATE EQUALS 6\% OF INPUT VOLUME _.... - FROM THIS STUDY

4. FOREST CITY WATER/SEWER COST EST $\$ 2.50 / 1000$

5. NACL COST EQUAL TO \$.035/ LB

6. POLYMER CHEM COST AT POTW EQUALS $\$ 1.20 / 1000 \cdots--B E T Z$

7. PYS/CHEM CHEM COST AT PLANT EQUALS $\$ 2.00 / 1000----$ FROM STUDY

8. SHARED LABOR COSTS BETWEEN UF/NF AND PHS/CHEM

\section{CALCULATIONS}

$(120,000 \mathrm{G} / \mathrm{D})^{*}(3.785 \mathrm{~L} / \mathrm{G})^{*}(20 \mathrm{~g} / \mathrm{L})^{*}(1 / 454 \mathrm{LB} / \mathrm{g})=20,009 \mathrm{LB} / \mathrm{D}$ OF NaCl

20,009 LB/D * $350 * .035=\$ 245,108$ BRINE COST SAVINGS

120 TG/D * $350 * \$ 2.50=\$ 105,000$ WATER COST SAVINGS

WWTP POLYMER COST FOR COLOR REMOVAL $=\$ 504,000$

(TREATMENT OF ENTIRE DISCHARGE AT POTW)

PROPOSED COLOR TREATMENT $=7200 * \$ 2.00 * 350=\$ 5040$

SLUDGE DISPOSAL COSTS $=\$ 4725$

$\$ 245,108+\$ 105,000+\$ 504,000-\$ 5040-\$ 4725$

OPERATING SAVINGS $=\$ 844,343$

ESTIMATED CAPITAL $=\$ 500,000$ (FROM COMPEDITIVE BIDDING OF PRESENT EQUIP)

PHYS/CHEM CAPITAL EST. $=\$ 80,000$

ESTIMATED MANTAINENCE $\$ 15,000$

LABOR COST $=\$ 70,000$

ENERGY COST $=\$ 15,000$

(SLUDGE DISPOSAL COST) @ $\$ 30.00 / T$

$7200 \mathrm{G} / \mathrm{D} * 1.5 \%$ SOLIDS * $8.34=900 \mathrm{LB} / \mathrm{D}$

$1200 * 350=210 \mathrm{~T} / \mathrm{N} * \$ 30.00=\$ 4725$

DEPRECIATION $=\$ 29,000$

OPERATIONAL COST $=\$ 29,000+\$ 15,000+\$ 15,000+\$ 80,000$ $=\$ 139,000$

PAYBACK $=\$ 580,000 /(\$ 844,343-\$ 139,000)=0.82$ YEARS

IF COLOR REMOVAL AT POTW IS NOT REQUIRED THEN

PAYBACK $=\$ 580,000 /(\$ 340,343-\$ 139,000)=2.9$ YEARS 

ULTRA-FILTER

OPERATIONAL DATA

\begin{tabular}{|c|c|c|c|c|c|}
\hline DATE $/ T I M E$ & psi IN & psi OUT & TEMP $F$ & PERM gpm & gal $\times 100$ \\
\hline $4 / 28-09: 45$ & 72 & 60 & 114 & 7.59 & 99 \\
\hline $4 / 28-10: 00$ & 72 & 60 & 114 & 7.39 & 100 \\
\hline $4 / 28-10: 30$ & 71 & 60 & 115 & 7.1 & 102 \\
\hline \multicolumn{6}{|l|}{ i } \\
\hline $4 / 29-08: 15$ & 72 & 60 & 109 & 5.23 & 104 \\
\hline $4 / 29-08: 35$ & 71 & 60 & 110 & 5.25 & 105 \\
\hline $4 / 29-10: 50$ & 70 & 57 & 111 & 5.3 & 107 \\
\hline $4 / 29-11: 18$ & 70 & 58 & 114 & 4.5 & 109 \\
\hline $4 / 29-11: 34$ & 69 & 58 & 115 & 4.5 & 110 \\
\hline $4 / 29-11: 40$ & 70 & 58 & 116 & 4.5 & 110 \\
\hline $4 / 29-11: 54$ & 68 & 57 & 116 & 4.5 & 111 \\
\hline $4 / 29-12: 10$ & 66 & 55 & 116 & 4.4 & 111 \\
\hline $4 / 29-12: 20$ & 66 & 55 & 116 & 4.4 & 112 \\
\hline 4/29-12:35 & 71 & 60 & 118 & 4.4 & 113 \\
\hline $4 / 29-12: 50$ & 70 & 60 & 117 & 4.3 & 113 \\
\hline & & & & & \\
\hline 5/1-09:00 & 69 & 58 & 104 & 4.8 & 116 \\
\hline $5 / 1-09: 35$ & 65 & 55 & 104 & 4.5 & 117 \\
\hline $5 / 1-10: 00$ & 70 & 60 & 105 & 4.5 & 118 \\
\hline $5 / 1-10: 10$ & 68 & 59 & 105 & 4.5 & 118 \\
\hline $5 / 1-10: 34$ & 65 & 56 & 105 & 4.3 & 119 \\
\hline $5 / 1-10: 55$ & 63 & 54 & 107 & 4.2 & 120 \\
\hline $5 / 1-11: 10$ & 62 & 53 & 107 & 4.1 & 121 \\
\hline $5 / 1-11: 27$ & 60 & 50 & 108 & 4 & 121 \\
\hline $5 / 1-11: 45$ & 57 & 49 & 108 & 3.9 & 122 \\
\hline & & & & & \\
\hline $5 / 2-09: 50$ & 60 & 50 & 100 & 3.7 & 124 \\
\hline $5 / 2-10: 10$ & 68 & 58 & 103 & 3.9 & 125 \\
\hline $5 / 2-10: 37$ & 65 & 55 & 104 & 3.6 & 126 \\
\hline $5 / 2-11: 10$ & 60 & 50. & 105 & 3.3 & 127 \\
\hline $5 / 2-11: 33$ & 58 & 49 & 105 & 3.2 & 128 \\
\hline $5 / 5-10: 05$ & 69 & 60 & 112 & 2.1 & 130 \\
\hline $5 / 5-10: 27$ & 69 & 60 & 112 & 2.1 & 131 \\
\hline $5 / 5-10: 48$ & 69 & 60 & 112 & 2.11 & 131 \\
\hline $5 / 5-11: 07$ & 72 & 65 & 111 & 2.28 & 132 \\
\hline $5 / 5-11: 47$ & 70 & 63 & 112 & 2.2 & 133 \\
\hline $5 / 5-13: 44$ & 64 & 55 & 115 & 2.2 & 135 \\
\hline & & & 103 & 207 & \\
\hline$\frac{311-07: 30}{50000}$ & 80 & 34 & 703 & 2.81 & \\
\hline 5/7-08:00 & 80 & 33. & 104 & 2.88 & 143 \\
\hline $5 / 7-08: 30$ & 80 & 33 & 106 & 3.13 & \\
\hline $5 / 7-08: 50$ & 80 & 33 & 108 & 3.04 & 144 \\
\hline $5 / 7-09: 30$ & 80 & 34 & 108 & 3.06 & 144 \\
\hline & & & & & \\
\hline $5 / 12-08: 37$ & 85 & 48 & 123 & 3.59 & 146 \\
\hline $5 / 12-08: 51$ & 85 & 49 & 122 & 3.18 & 146 \\
\hline 5/12-09:45 & 85 & 49 & 121 & 2.99 & 147 \\
\hline $5 / 12-10: 15$ & 85 & 49 & 120 & 3.08 & 148 \\
\hline
\end{tabular}


ULTRA-FILTER

OPERATIONAL DATA

\begin{tabular}{|c|c|c|c|c|c|}
\hline $5 / 12-10: 45$ & 85 & 49 & 121 & 3.27 & 148 \\
\hline $5 / 12-11: 45$ & 85 & 49 & 120 & 3.35 & 149 \\
\hline $5 / 12-12: 20$ & 85 & 49 & 119 & 3.34 & 150 \\
\hline $5 / 12-12: 50$ & 84 & 48 & 120 & 3.4 & 151 \\
\hline $5 / 12-13: 50$ & 84 & 48 & 121 & 3.6 & 153 \\
\hline & & & & & \\
\hline 5/13-07:15 & 87 & 55 & 110 & 3.7 & 154 \\
\hline $5 / 13-07: 45$ & 87 & 54 & 112 & 4.01 & 155 \\
\hline $5 / 13-08: 15$ & 87 & 54 & 112 & 4.05 & 156 \\
\hline $5 / 13-08: 45$ & 87 & 54 & 113 & 4.11 & 157 \\
\hline 5/13-09:15 & 87 & 55 & 114 & 4.55 & 158 \\
\hline $5 / 13-09: 45$ & 86 & 55 & 114 & 4.66 & 159 \\
\hline 5/13-10:15 & 90 & 58 & 116 & 4.84 & 160 \\
\hline $5 / 13-10: 45$ & 87 & 50 & 118 & 4.46 & 161 \\
\hline $5 / 13-11: 35$ & 86 & 50 & 119 & 4.6 & 163 \\
\hline $5 / 13-12: 30$ & 86 & 49 & 121 & 4.89 & 166 \\
\hline 5/13-13:15 & 86 & 50 & 123 & 4.78 & 168 \\
\hline 5/15-10:06 & 85 & 45 & 110 & 5.26 & 182 \\
\hline $5 / 15-10: 45$ & 85 & 45 & 112 & 5.1 & 184 \\
\hline $5 / 15-11: 06$ & 85 & 45 & 113 & 5.22 & 185 \\
\hline $5 / 15-11: 30$ & 85 & 50 & 114 & 5.1 & 186 \\
\hline $5 / 15-12: 50$ & 85 & 50 & 119 & 5.4 & 190 \\
\hline $5 / 15-13: 25$ & 84 & 50 & 120 & 5.3 & 192 \\
\hline 5/15-13:50 & 84 & 50 & 120 & 5.57 & 193 \\
\hline $5 / 16-07: 15$ & 85 & 48 & 108 & 5.05 & 194 \\
\hline $5 / 16-08: 15$ & 85 & 51 & 112 & 4.58 & 197 \\
\hline $5 / 16-08: 45$ & 85 & 51 & 113 & 4.81 & 198 \\
\hline $5 / 16-09: 45$ & 84 & 47 & 113 & 4.85 & 199 \\
\hline 5/19-07:15 & 85 & 44 & 90 & 4.18 & 202 \\
\hline $5 / 19-07: 45$ & 85 & 45 & 93 & 3.93 & 203 \\
\hline $5 / 19-08: 20$ & 85 & 45 & 94 & 3.79 & 205 \\
\hline 5/19-08:50 & 85 & 46 & 96 & 3.76 & 206 \\
\hline $5 / 19-10: 15$ & 85 & 47 & 97 & 4.13 & 206 \\
\hline $5 / 19-10: 48$ & 85 & 47 & 100 & 3.9 & 208 \\
\hline $5 / 19-11: 40$ & 85 & 49 & 101 & 4.24 & 209 \\
\hline 5/19-12:20 & 85 & 49 & 108 & 4.23 & 210 \\
\hline 5/19_13:20 & 86 & 52 & 111 & 4.81 & 212 \\
\hline 5/19-13:55 & 86 & 52 & 114 & 4.92 & 213 \\
\hline $5 / 20-07: 15$ & 86 & 45 & 107 & 4.16 & 214 \\
\hline $5 / 20-07: 45$ & 86 & 46 & 108 & 4.5 & 215 \\
\hline 5/20-08:15 & 85 & 46 & 110 & 4.25 & 216 \\
\hline $5 / 20-08: 45$ & 85 & 46 & 113 & 4.25 & 218 \\
\hline $5 / 20-09: 15$ & 85 & 46 & 115 & 4.28 & 219 \\
\hline $5 / 20-09: 45$ & 85 & 46 & 117 & 4.49 & 221 \\
\hline $5 / 20-10: 15$ & 84 & 45 & 117 & 4.2 & 222 \\
\hline $5 / 20-10: 45$ & 84 & 45 & 117 & 4.36 & 223 \\
\hline $5 / 20-11: 45$ & 86 & 52 & 117 & 4.59 & 224 \\
\hline
\end{tabular}


ULTRA-FILTER

OPERATIONAL DATA

\begin{tabular}{|c|c|c|c|c|c|}
\hline $5 / 20-12: 15$ & 86 & 52 & 117 & 4.64 & 225 \\
\hline $5 / 20-12: 45$ & 86 & 52 & 119 & 4.84 & 227 \\
\hline $5 / 20-14: 00$ & 86 & 52 & 119 & 4.65 & 229 \\
\hline & & & & 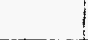 & \\
\hline $5 / 21-07: 15$ & 86 & 51 & 108 & 4.5 & 230 \\
\hline $5 / 21-07: 45$ & 86 & 51 & 110 & 4.29 & 232 \\
\hline $5 / 21-08: 15$ & 86 & 51 & 111 & 4.25 & 233 \\
\hline $5 / 21-08: 45$ & 86 & 51 & 112 & 4.57 & 234 \\
\hline $5 / 21-09: 45$ & 86 & 52 & 112 & 4.39 & 235 \\
\hline $5 / 22-09: 30$ & 85 & 51 & 112 & 5.21 & 239 \\
\hline $5 / 22-10: 15$ & 85 & 51 & 113 & 5.05 & 241 \\
\hline $5 / 22-10: 45$ & 85 & 51 & 114 & 5.12 & 242 \\
\hline $5 / 22-11: 20$ & 85 & 51 & 115 & 5.05 & 244 \\
\hline $5 / 22-11: 45$ & 85 & 51 & 117 & 5.08 & 245 \\
\hline $5 / 22-12: 15$ & 85 & 51 & 120 & 5.32 & 247 \\
\hline $5 / 22-12: 45$ & 85 & 51 & 121 & 5.13 & 248 \\
\hline $5 / 22-14: 00$ & 85 & 51 & 124 & 5.17 & 252 \\
\hline $5 / 23-07: 30$ & 85 & 49 & 112 & 4.67 & 254 \\
\hline $5 / 23-08: 10$ & 85 & 50 & 115 & 4.87 & 255 \\
\hline $5 / 23-08: 45$ & 85 & 50 & 118 & 4.71 & 257 \\
\hline $5 / 23-09: 15$ & 85 & 50 & 120 & 4.97 & 259 \\
\hline $5 / 23-09: 55$ & 84 & 50 & 121 & 5.07 & 260 \\
\hline $5 / 23-10: 30$ & 84 & 50 & 121 & 5.25 & 263 \\
\hline $5 / 28-10: 15$ & 84 & 40 & 96 & 4.6 & 275 \\
\hline $5 / 28-10: 45$ & 84 & 40 & 96 & 4.3 & 276 \\
\hline $5 / 28-11: 20$ & 84 & 40 & 98 & 4.36 & 278 \\
\hline $5 / 28-11: 45$ & 84 & 40 & 99 & 4.45 & 279 \\
\hline $5 / 28-12: 15$ & 83 & 40 & 100 & 4.64 & 280 \\
\hline $5 / 28-12: 45$ & 82 & 40 & 104 & 4.35 & 281 \\
\hline $5 / 28-13: 15$ & 82 & 40 & 104 & 4.25 & 281 \\
\hline & & & & & \\
\hline$\frac{5 / 29-09: 20}{5 / 29-09: 45}$ & $\frac{90}{89}$ & $\frac{60}{56}$ & $\frac{113}{117}$ & $\begin{array}{l}7.58 \\
7.13\end{array}$ & $\frac{283}{285}$ \\
\hline $5 / 29-10: 15$ & 88 & 56 & 121 & 7.06 & 287 \\
\hline $5 / 29-10: 45$ & 88 & 56 & 121 & 7.32 & 289 \\
\hline $5 / 29-11: 15$ & 89 & 59 & 117 & 6.52 & 292 \\
\hline $5 / 29-11: 45$ & 89 & 58 & 116 & 6.58 & 293 \\
\hline $5 / 29-12: 15$ & 88 & 58 & 117 & 6.52 & 295 \\
\hline $5 / 29-12: 45$ & 88 & 58 & 118 & 6.56 & 297 \\
\hline $5 / 29-13: 15$ & 88 & 58 & 118 & 6.65 & 299 \\
\hline $5 / 29-13: 45$ & 88 & 58 & 118 & 6.5 & 301 \\
\hline $5 / 30-07: 30$ & 92 & 58 & 108 & $6 . \overline{41}$ & 303 \\
\hline $5 / 30-07: 45$ & 90 & 56 & 108 & 6 & 304 \\
\hline $5 / 30-08: 15$ & $9 \overline{0}$ & 56 & 109 & 5.77 & 305 \\
\hline $5 / 30-08: 45$ & 90 & 55 & 113 & 5.85 & 307 \\
\hline $5 / 30-09: 45$ & 88 & 54 & 114 & 5.68 & 309 \\
\hline $5 / 30-10: 15$ & 88 & 54 & 114 & 5.4 & 310 \\
\hline
\end{tabular}


ULTRA-FILTER

OPERATIONAL DATA

\begin{tabular}{|c|c|c|c|c|c|}
\hline $5 / 30-10: 45$ & 88 & 54 & 116 & 5.34 & 312 \\
\hline $5 / 30-11: 45$ & 88 & 54 & 118 & 4.71 & 315 \\
\hline $5 / 30-12: 15$ & 88 & 53 & 120 & 5.18 & 317 \\
\hline $5 / 30-12: 45$ & 87 & 53 & 121 & 5.06 & 318 \\
\hline $5 / 30-13: 15$ & 86 & 53 & 122 & 4.78 & 320 \\
\hline $5 / 30-13: 45$ & 86 & 53 & 122 & 5.18 & 320 \\
\hline $6 / 2-08: 45$ & 90 & 60 & 109 & 5.92 & 321 \\
\hline $6 / 2-09: 15$ & 88 & 57 & 110 & 5.53 & 322 \\
\hline $6 / 2-09: 45$ & 88 & 57 & 111 & 5.28 & 323 \\
\hline $6 / 2-10: 15$ & 90 & 60 & 112 & 5.35 & 324 \\
\hline $6 / 2-10: 45$ & 90 & 60 & 112 & 5.01 & 326 \\
\hline $6 / 2-11: 15$ & 90 & 60 & 113 & 5.01 & 328 \\
\hline $6 / 2-11: 45$ & 90 & 60 & 116 & 5.32 & 329 \\
\hline $6 / 2-12: 15$ & 90 & 60 & 118 & 5.25 & 331 \\
\hline $6 / 2-12: 45$ & 90 & 60 & 119 & 5.35 & 332 \\
\hline $6 / 2-13: 15$ & 89 & 60 & 121 & 5.36 & 334 \\
\hline $6 / 2-13: 45$ & 88 & 60 & 122 & 5.45 & 335 \\
\hline $6 / 3-07: 45$ & 90 & 56 & 110 & 4.96 & 338 \\
\hline $6 / 3-08: 15$ & 90 & 56 & 112 & 4.7 & 339 \\
\hline $6 / 3-08: 45$ & 90 & 56 & 112 & 4.53 & 341 \\
\hline $6 / 3-09: 15$ & 90 & 55 & 112 & 4.52 & 341 \\
\hline $6 / 3-09: 45$ & 89 & 55 & 112 & 4.48 & 342 \\
\hline $6 / 3-10: 15$ & 88 & 55 & 112 & 4.52 & 344 \\
\hline $6 / 3-10: 45$ & 88 & 55 & 113 & 4.44 & 345 \\
\hline $6 / 3-11: 15$ & 88 & 55 & 113 & 4.53 & 347 \\
\hline $6 / 3-11: 45$ & 88 & 55 & 114 & 4.46 & 347 \\
\hline $6 / 3-12: 15$ & 88 & 55 & 114 & 4.48 & 349 \\
\hline $6 / 3-12: 45$ & 88 & 54 & 115 & 4.43 & 350 \\
\hline $6 / 3-13: 15$ & 88 & 54 & 117 & 4.9 & 352 \\
\hline $6 / 3-13: 45$ & 88 & 54 & 118 & 4.68 & 353 \\
\hline $6 / 4-07: 15$ & 85 & 47 & 108 & 3.64 & 354 \\
\hline $6 / 4-07: 45$ & 85 & 48 & 109 & 3.35 & 355 \\
\hline $6 / 4-08: 15$ & 85 & 48 & 110 & 3.32 & 356 \\
\hline 6/4-08:45 & 85 & 48 & 111 & 3.5 & 357 \\
\hline $6 / 4-09: 15$ & 84 & 48 & 112 & 3.34 & 358 \\
\hline $6 / 4-09: 45$ & 84 & 49 & 112 & 3.33 & 359 \\
\hline $6 / 4-10: 15$ & 84 & 49 & 114 & 3.28 & 359 \\
\hline $6 / 4-10: 45$ & 85 & 51 & 117 & 3.35 & 360 \\
\hline $6 / 4-11: 45$ & 85 & 52 & 116 & 3.05 & 361 \\
\hline $6 / 4-12: 45$ & 85 & 52 & 117 & 3.2 & 362 \\
\hline $6 / 5-08: 50$ & 89 & 55 & 107 & 3.07 & 362 \\
\hline $6 / 5-09: 15$ & 89 & 55 & 112 & 3.46 & 263 \\
\hline $6 / 5-09: 45$ & 92 & 60 & 114 & 4.09 & 364 \\
\hline $6 / 5-10: 15$ & 91 & 60 & 115 & 4.02 & 366 \\
\hline $6 / 5-10: 45$ & 90 & 60 & 121 & 4.74 & 367 \\
\hline $6 / 5-11: 15$ & 90 & 60 & 122 & 4.66 & 368 \\
\hline $6 / 5-11: 45$ & 90 & 60 & 122 & 4.59 & 370 \\
\hline
\end{tabular}


ULTRA-FILTER

OPERATIONAL DATA

\begin{tabular}{|l|r|r|r|r|r|}
\hline $6 / 5-12: 15$ & 90 & 60 & 122 & 4.38 & 371 \\
\hline $6 / 5-12: 45$ & 90 & 60 & 123 & 4.12 & 372 \\
\hline $6 / 5-13: 15$ & 89 & 60 & 124 & 4.27 & 373 \\
\hline $6 / 5-13: 45$ & 88 & 60 & 124 & 4.04 & 375 \\
\hline & & & & & \\
\hline $6 / 6-07: 15$ & 90 & 60 & 112 & 2.72 & 376 \\
\hline $6 / 6-07: 45$ & 90 & 60 & 113 & 2.9 & 376 \\
\hline $6 / 6-08: 45$ & 88 & 60 & 114 & 2.88 & 378 \\
\hline $6 / 6-09: 15$ & 88 & 60 & 115 & 3.07 & 379 \\
\hline $6 / 6-11: 45$ & 87 & 60 & 112 & 2.86 & 379 \\
\hline $6 / 6-12: 15$ & 92 & 60 & 113 & 3.6 & 380 \\
\hline $6 / 6-12: 45$ & 92 & 50 & 114 & 3.46 & 381 \\
\hline & & & & & \\
\hline $6 / 9-09: 30$ & 88 & 52 & 114 & 4.18 & 381 \\
\hline $6 / 9-10: 00$ & 88 & 52 & 116 & 4.91 & 383 \\
\hline $6 / 9-10: 30$ & 87 & 51 & 117 & 5.49 & 384 \\
\hline $6 / 9-11: 30$ & 86 & 50 & 116 & 5.63 & 386 \\
\hline $6 / 9-12: 00$ & 86 & 50 & 116 & 5.45 & 387 \\
\hline $6 / 9-12: 30$ & 86 & 50 & 117 & 5.95 & 389 \\
\hline $6 / 9-12: 55$ & 86 & 50 & 118 & 5.52 & 390 \\
\hline $6 / 9-13: 30$ & 85 & 50 & 120 & 6.05 & 393 \\
\hline $6 / 9-14: 00$ & 85 & 50 & 120 & 5.86 & 394 \\
\hline $6 / 10-07: 15$ & 90 & 60 & 109 & 6.1 & 396 \\
\hline $6 / 10-08: 15$ & 90 & 60 & 114 & 6.22 & 399 \\
\hline $6 / 10-08: 45$ & 90 & 60 & 114 & 6.25 & 401 \\
\hline $6 / 10-09: 15$ & 90 & 60 & 114 & 6.3 & 403 \\
\hline $6 / 10-09: 45$ & 89 & 60 & 114 & 5.94 & 405 \\
\hline $6 / 10-10: 15$ & 88 & 60 & 116 & 5.86 & 408 \\
\hline $6 / 10-10: 45$ & 88 & 60 & 117 & 5.8 & 409 \\
\hline $6 / 10-11: 15$ & 88 & 60 & 117 & 6.19 & 411 \\
\hline $6 / 10-11: 45$ & 88 & 60 & 117 & 6.34 & 412 \\
\hline $6 / 10-12: 30$ & 88 & 60 & 117 & 5.94 & 415 \\
\hline $6 / 10-12: 45$ & 88 & 60 & 119 & 6.14 & 416 \\
\hline $6 / 10-13: 25$ & 86 & 59 & 120 & 5.91 & 418 \\
\hline $6 / 10-14: 00$ & 86 & 59 & 122 & 5.8 & 420 \\
\hline $6 / 11-07: 30$ & 85 & 44 & 108 & 4.66 & 422 \\
\hline $6 / 11-08: 00$ & 85 & 45 & 110 & 4.53 & 424 \\
\hline $6 / 11-08: 30$ & 85 & 45 & 110 & 4.3 & 425 \\
\hline $6 / 11-09: 45$ & 85 & 47 & 112 & 4.23 & 426 \\
\hline $6 / 11-10: 15$ & 85 & 47 & 114 & 4.24 & 428 \\
\hline $6 / 11-10: 45$ & 85 & 47 & 115 & 4.27 & 429 \\
\hline $6 / 11-11: 15$ & 85 & 47 & 116 & 4.28 & 431 \\
\hline $6 / 11-11: 45$ & 85 & 47 & 117 & 4.31 & 431 \\
\hline $6 / 11-13: 15$ & $87.07: 45$ & 47 & 121 & 4.31 & 434 \\
\hline & 90 & 60 & 110 & 4.32 & 436 \\
\hline & & 60 & 112 & 4.42 & 446 \\
\hline
\end{tabular}


ULTRA-FILTER

OPERATIONAL DATA

\begin{tabular}{|c|c|c|c|c|c|}
\hline $6 / 13-08: 45$ & 90 & 60 & 112 & 4.45 & 448 \\
\hline $6 / 13-09: 15$ & 90 & 60 & 114 & 4.44 & 449 \\
\hline $6 / 13-09: 45$ & 90 & 60 & 115 & 4.27 & 450 \\
\hline $6 / 13-10: 15$ & 89 & 60 & 115 & 4.26 & 451 \\
\hline $6 / 13-10: 45$ & 89 & 60 & 117 & 4.28 & 453 \\
\hline $6 / 13-11: 15$ & 89 & 60 & 120 & 4.57 & 454 \\
\hline $6 / 13-11: 45$ & 89 & 60 & 120 & 4.47 & 456 \\
\hline$6 \longdiv { 1 3 - 1 2 : 1 5 }$ & $\overline{89}$ & 60 & 120 & 4.28 & 457 \\
\hline $6 / 13-12: 45$ & 88 & 60 & 119 & 4.12 & 458 \\
\hline$6 \longdiv { 1 3 - 1 3 : 1 5 }$ & 88 & 60 & 120 & 4.13 & 459 \\
\hline $6 / 13-13: 45$ & 88 & 60 & 121 & 4.11 & 461 \\
\hline $6 / 16-07: 40$ & 88 & 50 & 100 & 324 & 461 \\
\hline $6 / 16-08: 15$ & 88 & 50 & 104 & 2.94 & 463 \\
\hline $6 / 16-08: 45$ & 92 & 60 & 106 & 3.35 & 463 \\
\hline $6 / 16-09: 15$ & 90 & 60 & 107 & 3.14 & 464 \\
\hline $6 / 16-09: 45$ & 90 & 60 & 108 & 3.17 & 465 \\
\hline $6 / 16-10: 15$ & 90 & 60 & 109 & 3.15 & 466 \\
\hline $6 / 16-10: 45$ & 90 & 60 & 110 & 3.12 & 467 \\
\hline $6 / 16-11: 15$ & 90 & 60 & 116 & 3.16 & 468 \\
\hline $6 / 16-11: 45$ & 90 & 60 & 113 & 3.38 & 469 \\
\hline $6 / 16-12: 15$ & 90 & 60 & 114 & 3.16 & 470 \\
\hline $6 / 16-12: 45$ & 90 & 60 & 114 & 3.22 & 471 \\
\hline $6 / 16-13: 15$ & 90 & 60 & 115 & 3.46 & 472 \\
\hline $6 / 17-08: 30$ & 88 & 51 & 111 & 2.79 & 474 \\
\hline $6 / 17-08: 45$ & 92 & 60 & 112 & 3.59 & 474 \\
\hline $6 / 17-09: 15$ & 92 & 60 & 112 & 3.71 & 476 \\
\hline $6 / 17-09: 45$ & 92 & 60 & 112 & 3.95 & 477 \\
\hline $6 / 17-10: 15$ & 91 & 60 & 112 & 3.79 & 478 \\
\hline $6 / 17-10: 45$ & 91 & 60 & 113 & 4.17 & 479 \\
\hline $6 / 17-11: 15$ & 91 & 60 & 114 & 4.4 & 481 \\
\hline $6 / 17-11: 45$ & 90 & 60 & 115 & 4.25 & 482 \\
\hline $6 / 17-12: 15$ & 90 & 60 & 118 & 4.4 & 483 \\
\hline $6 / 17-12: 45$ & 90 & 60 & 119 & 4.68 & 485 \\
\hline $6 / 17-13: 15$ & 90 & 60 & 126 & 3.39 & 486 \\
\hline $6 / 17-13: 45$ & 90 & 60 & 127 & 1.93 & 487 \\
\hline $6 / 18-07: 15$ & 90 & 59 & 113 & 1.28 & 487 \\
\hline $6 / 18-07: 45$ & 90 & 59 & 112 & 1.36 & 487 \\
\hline $6 / 18-08: 15$ & 90 & 60 & 113 & 1.55 & 488 \\
\hline $6 / 18-08: 45$ & 90 & 60 & 114 & 1.94 & 488 \\
\hline $6 / 18-09: 15$ & 90 & 60 & 118 & 3.37 & 489 \\
\hline $6 / 18-09: 45$ & 90 & 60 & 119 & 3.71 & 490 \\
\hline $6 / 18-10: 15$ & 90 & 60 & 119 & 3.67 & 491 \\
\hline $6 / 18-10: 45$ & 90 & 60 & 120 & 3.38 & 492 \\
\hline $6 / 18-11: 15$ & 89 & 60 & 121 & 3.46 & $4 \overline{93}$ \\
\hline $6 / 18-11: 45$ & 89 & 59 & 122 & 3.33 & 494 \\
\hline $6 / 18-12: 15$ & 88 & 59 & 122 & 3.2 & 495 \\
\hline $6 / 18-12: 45$ & 88 & 59 & 122 & 3.29 & 496 \\
\hline $6 / 18-13: 15$ & 88 & 59 & 124 & 3.35 & 497 \\
\hline
\end{tabular}


ULTRA-FILTER

OPERATIONAL DATA

\begin{tabular}{|c|c|c|c|c|c|}
\hline $6 / 18-13: 45$ & 88 & 59 & 124 & 3.32 & 497 \\
\hline $6 / 19-07: 30$ & 90 & 60 & 110 & 2.29 & 498 \\
\hline $6 / 19-07: 45$ & 90 & 60 & 110 & 2.66 & 498 \\
\hline $6 / 19-08: 15$ & 90 & 60 & 111 & 2.78 & 499 \\
\hline $6 / 19-09: 15$ & 90 & 58 & 111 & 2.99 & 499 \\
\hline $6 / 19-09: 45$ & 90 & 59 & 114 & 3.27 & 500 \\
\hline $6 / 19-10: 15$ & 90 & 59 & $\overline{119}$ & 3.34 & 501 \\
\hline $6 / 19-10: 45$ & 90 & 59 & 120 & 3.59 & 502 \\
\hline $6 / 19-11: 15$ & 90 & 58 & 122 & 3.6 & 503 \\
\hline $6 / 19-11: 45$ & 90 & 58 & 122 & 3.7 & 504 \\
\hline $6 / 19-12: 45$ & 89 & 58 & 120 & 3.73 & 505 \\
\hline $6 / 19-13: 15$ & 88 & 57 & 120 & 3.64 & 506 \\
\hline $6 / 19-13: 45$ & 88 & 57 & 120 & 3.44 & 507 \\
\hline & & & & & \\
\hline $6 / 20-07: 15$ & 91 & 60 & 108 & 3 & 508 \\
\hline $6 / 20-07: 45$ & 91 & 60 & 110 & 3.09 & 509 \\
\hline $6 / 20-08: 15$ & 91 & 60 & 112 & 3.1 & 510 \\
\hline $6 / 20-08: 45$ & 91 & 60 & 112 & 3.11 & 511 \\
\hline $6 / 20-09: 15$ & 88 & 56 & 112 & 3.08 & 511 \\
\hline $6 / 20-09: 45$ & 88 & 56 & 113 & 2.84 & 512 \\
\hline $6 / 20-10: 15$ & 87 & 56 & 114 & 3.09 & 513 \\
\hline $6 / 20-11: 15$ & 88 & 57 & 112 & 3.15 & 513 \\
\hline $6 / 20-11: 45$ & 88 & 57 . & 112 & 2.97 & 514 \\
\hline $6 / 20-12: 15$ & 87 & 57 & 112 & 3.13 & 515 \\
\hline $6 / 20-12: 45$ & 87 & 57 & 112 & 3.15 & 516 \\
\hline & & & & & \\
\hline 6/24-07:30 & 90 & 54 & 104 & 5.89 & 523 \\
\hline $6 / 24-07: 45$ & 88 & 53 & 106 & 5.8 & 524 \\
\hline $6 / 24-08: 15$ & 88 & 53 & 108 & 6 & 526 \\
\hline $6 / 24-08: 45$ & 87 & 53 & 110 & 6.56 & 528 \\
\hline 6/24-09:15 & 86 & 52 & 110 & 6.89 & 530 \\
\hline $6 / 24-09: 45$ & 86 & 52 & 111 & 7.12 & 532 \\
\hline $6 / 24-10: 15$ & 86 & 52 & 112 & 7.78 & 534 \\
\hline $6 / 24-10: 45$ & 85 & 52 & 114 & 7.88 & 536 \\
\hline $6 / 24-12: 45$ & 84 & 51 & 117 & 8.35 & 541 \\
\hline $6 / 24-13: 15$ & 83 & 50 & 118 & 8.57 & 544 \\
\hline $6 / 25-09: 23$ & 90 & 57 & 106 & 8.22 & 547 \\
\hline $6 / 25-10: 07$ & 89 & 56 & 108 & 8.26 & 550 \\
\hline $6 / 25-10: 25$ & 89 & 56 & 110 & 8.33 & 552 \\
\hline $6 / 25-10: 45$ & 88 & 56 & 110 & 8.83 & 553 \\
\hline $6 / 25-11: 30$ & 88 & 56 & 112 & 8.47 & 557 \\
\hline $6 / 25-13: 40$ & 90 & 64 & 111 & 8.32 & 560 \\
\hline $6 / 25-14: 20$ & 89 & 57 & 112 & 8.3 & 564 \\
\hline $6 / 26-07: 45$ & 90 & 60 & 109 & 9.01 & 579 \\
\hline $6 / 26-08: 15$ & 89 & 60 & 112 & 8.83 & 582 \\
\hline $6 / 26-08: 45$ & 89 & 60 & 114 & 8.58 & 584 \\
\hline $6 / 26-09: 45$ & 88 & 60 & 114 & 8.47 & 587 \\
\hline $6 / 26-11: 15$ & 88 & 60 & 116 & 8.29 & 591 \\
\hline
\end{tabular}


ULTRA-FILTER

OPERATIONAL DATA

\begin{tabular}{|c|c|c|c|c|c|}
\hline $6 / 26-12: 15$ & 88 & 60 & 121 & 8.93 & 595 \\
\hline $6 / 26-13: 15$ & 88 & $5 \overline{9}$ & 122 & 8.48 & 597 \\
\hline $6 / 30-07: 15$ & 90 & 52 & 88 & 6.53 & 598 \\
\hline $6 / 30-07: 45$ & 88 & 52 & 91 & 6.66 & 600 \\
\hline $6 / 30-08: 15$ & 88 & 51 & 98 & 7.11 & 602 \\
\hline $6 / 30-08: 45$ & 86 & 51 & 100 & 7.24 & 603 \\
\hline $6 / 30-09: 45$ & 86 & 50 & 101 & 7.37 & 605 \\
\hline $6 / 30-10: 15$ & 85 & 50 & 104 & 7.76 & 607 \\
\hline $6 / 30-10: 45$ & 85 & 49 & 107 & 7.43 & 609 \\
\hline $6 / 30-11: 45$ & 85 & 50 & 105 & 7.76 & 611 \\
\hline $6 / 30-12: 15$ & 84 & 49 & 107 & 7.8 & 613 \\
\hline $6 / 30-12: 45$ & 84 & 48 & 108 & 7.61 & 616 \\
\hline $6 / 30-13: 15$ & 84 & 48 & 108 & 8.04 & 617 \\
\hline & & & & & \\
\hline $7 / 1-07: 20$ & 92 & 60 & 102 & 8.7 & 618 \\
\hline $7 / 1-07: 45$ & 90 & 60 & 104 & 8.32 & 620 \\
\hline $7 / 1-08: 45$ & 90 & 60 & 108 & 9.29 & 626 \\
\hline $7 / 1-09: 45$ & 89 & 60 & 108 & 9.08 & 629 \\
\hline $7 / 1-10: 15$ & 88 & 60 & 110 & 9.24 & 631 \\
\hline $7 / 1-10: 45$ & 88 & 60 & 111 & 9.05 & 634 \\
\hline $7 / 1-11: 45$ & 87 & 60 & 111 & 8.88 & 635 \\
\hline $7 / 1-12: 15$ & 86 & 60 & 110 & 8.79 & 638 \\
\hline $7 / 1-12: 45$ & 85 & 60 & 110 & 8.85 & 640 \\
\hline $7 / 1-13: 30$ & 85 & 60 & 110 & 8.4 & 641 \\
\hline $7 / 2-07: 45$ & 88 & 49 & 98 & 8.79 & 642 \\
\hline $7 / 2-08: 15$ & 86 & 49 & 105 & 8.38 & 645 \\
\hline $7 / 2-08: 45$ & 85 & 49 & 108 & 8.61 & 647 \\
\hline $7 / 2-10: 15$ & 93 & 45 & 110 & 9.93 & 652 \\
\hline $7 / 2-10: 45$ & 92 & 45 & 109 & 9.42 & 655 \\
\hline $7 / 2-11: 15$ & 92 & 45 & 109 & 9.58 & 657 \\
\hline $7 / 2-12: 15$ & 89 & 60 & 109 & 9.65 & 659 \\
\hline $7 / 2-12: 45$ & 88 & 60 & 110 & 9.41 & 661 \\
\hline & & & & & \\
\hline $7 / 8-11: 20$ & 88 & 57 & 112 & 7.49 & 662 \\
\hline $7 / 8-11: 45$ & 87 & 60 & 117 & 8.67 & 664 \\
\hline $7 / 8-12: 15$ & 86 & 59 & 117 & 8.25 & 667 \\
\hline $7 / 8-12: 45$ & 85 & 58 & 116 & 8.77 & 669 \\
\hline $7 / 8-13: 15$ & 90 & 60 & 114 & 9.2 & 669 \\
\hline $7 / 8-13: 45$ & 88 & 60 & 115 & 8.63 & 672 \\
\hline 7007.15 & 07 & 60 & 101 & 021 & 675 \\
\hline $7 / 9-07: 45$ & 92 & 60 & 106 & 9.46 & 677 \\
\hline $7 / 9-08: 15$ & 92 & 60 & 108 & 8.14 & 679 \\
\hline $7 / 9-08: 45$ & 90 & 60 & 109 & 8.32 & 682 \\
\hline $7 / 9-09: 15$ & 90 & 60 & 109 & 7.92 & 682 \\
\hline $7 / 9-09: 45$ & 90 & 60 & 111 & 8.93 & 684 \\
\hline $7 / 9-10: 15$ & 90 & 60 & 112 & 8.64 & 687 \\
\hline $7 / 9-10: 45$ & 89 & 60 & 114 & 8.8 & 689 \\
\hline $7 / 9-11: 45$ & 86 & 60 & 115 & 8.39 & 692 \\
\hline
\end{tabular}


ULTRA-FILTER

OPERATIONAL DATA

\begin{tabular}{|c|c|c|c|c|c|}
\hline $7 / 9-12: 15$ & 90 & 60 & 117 & 9.08 & 695 \\
\hline $7 / 9-12: 45$ & 90 & 60 & 117 & 8.03 & 697 \\
\hline $7 / 10-08: 00$ & 90 & 53 & 108 & 7.48 & 699 \\
\hline $7 / 10-08: 15$ & 89 & 53 & 108 & 7.3 & 700 \\
\hline $7 / 10-08: 45$ & 88 & 52 & 110 & 7.64 & 702 \\
\hline $7 / 10-09: 15$ & 88 & 52 & 110 & 7.76 & 703 \\
\hline $7 / 10-09: 45$ & 88 & 52 & 111 & 7.91 & 704 \\
\hline $7 / 10-10: 15$ & 88 & 52 & 112 & 7.6 & 707 \\
\hline $7 / 10-10: 45$ & 88 & 52 & 113 & 7.66 & 709 \\
\hline $7 / 10-11: 15$ & 88 & 52 & 112 & 7.51 & 710 \\
\hline $7 / 10-11: 45$ & 88 & 52 & 114 & 7.81 & 711 \\
\hline $7 / 10: 12: 15$ & 87 & 52 & 115 & 7.9 & 713 \\
\hline & & & & & \\
\hline 7/111-07:15 & 90 & 58 & 108 & 7.23 & 714 \\
\hline $7 / 11-07: 45$ & 90 & 58 & 108 & 7 & 716 \\
\hline $7 / 11-08: 15$ & 90 & 58 & 109 & 7.07 & 718 \\
\hline $7 / 11-08: 45$ & 88 & 57 & 110 & 6.98 & 721 \\
\hline $7 / 11-09: 15$ & 88 & 57 & 110 & 7 & 721 \\
\hline $7 / 11-09: 45$ & 88 & 56 & 110 & 7.25 & 723 \\
\hline $7 / 11-10: 15$ & 86 & 55 & 112 & 7.77 & 725 \\
\hline $7 / 11-10: 45$ & 86 & 55 & 112 & 7.83 & 727 \\
\hline $7 / 11-11: 45$ & 86 & 55 & 112 & 7.28 & 728 \\
\hline $7 / 11-12: 15$ & 86 & 55 & 114 & 7.43 & 731 \\
\hline $7 / 11-12: 45$ & 84 & 53 & 115 & 7.05 & 733 \\
\hline $7 / 14-11: 45$ & 88 & 52 & 101 & 6.04 & 736 \\
\hline $7 / 14-12: 15$ & 88 & 55 & 112 & 7.2 & 738 \\
\hline $7 / 14-12: 45$ & 86 & 54 & 113 & 7.17 & 741 \\
\hline $7 / 14-13: 15$ & 90 & 60 & 114 & 8.2 & 741 \\
\hline $7 / 14-13: 45$ & 90 & 60 & 114 & 7.82 & 743 \\
\hline $7 / 14-14: 15$ & 90 & 60 & 114 & 7.7 & 744 \\
\hline $7 / 15-07: 15$ & 90 & 60 & 106 & 8.55 & 746 \\
\hline $7 / 15-07: 45$ & 90 & 60 & 108 & 8.6 & 749 \\
\hline $7 / 15-08: 15$ & 90 & 60 & 110 & 8.85 & 751 \\
\hline $7 / 15-08: 45$ & 90 & 60 & 111 & 9.25 & 754 \\
\hline $7 / 15-09: 15$ & 90 & 60 & 114 & 9.33 & 754 \\
\hline $7 / 15-09: 45$ & 90 & 60 & 116 & 9.31 & 756 \\
\hline $7 / 15-10: 15$ & 90 & 60 & 118 & 9.45 & 760 \\
\hline $7 / 15-10: 45$ & 90 & 60 & 120 & 9.92 & 762 \\
\hline $7 / 15-11: 15$ & 89 & 60 & 120 & 10.79 & 763 \\
\hline $7 / 15-11: 45$ & 90 & 60 & 121 & 10.43 & 765 \\
\hline $7 / 15-12: 15$ & 90 & 60 & 124 & 9.93 & 768 \\
\hline $7 / 15-12: 45$ & 89 & 60 & 124 & 9.97 & 770 \\
\hline $7 / 15-13: 15$ & 88 & 60 & 122 & 10.73 & 770 \\
\hline $7 / 16-07: 45$ & 90 & 41 & 113 & 9.51 & 773 \\
\hline $7 / 16-08: 15$ & 90 & 44 & 115 & 8.56 & 775 \\
\hline $7 / 16-08: 45$ & 90 & 46 & 117 & 8.29 & 778 \\
\hline $7 / 16-09: 15$ & 89 & 55 & 117 & 8.11 & 778 \\
\hline
\end{tabular}


ULTRA-FILTER

OPERATIONAL DATA

\begin{tabular}{|l|r|r|r|r|r|}
\hline $7 / 16-09: 45$ & 89 & 55 & 121 & 8.47 & 780 \\
\hline $7 / 16-10: 15$ & 88 & 55 & 122 & 9.23 & 782 \\
\hline $7 / 16-10: 45$ & 88 & 54 & 122 & 9.83 & 786 \\
\hline $7 / 16-11: 45$ & 90 & 60 & 122 & 10.61 & 787 \\
\hline $7 / 16-12: 15$ & 90 & 60 & 122 & 10.25 & 791 \\
\hline $7 / 16-12: 45$ & 90 & 60 & 122 & 9.67 & 793 \\
\hline $7 / 16-13: 15$ & 90 & 60 & 122 & 9.48 & 794 \\
\hline
\end{tabular}


NANO-FILTER

OPERATIONAL DATA

\begin{tabular}{|c|c|c|c|c|c|c|}
\hline DATE-TIME & TEMP & PUMP psi & PRE & CONC & CONC & PERM \\
\hline & & & & & & \\
\hline $5 / 15-10: 55$ & 98 & 110 & 56 & 80 & & $40+$ \\
\hline $5 / 15-11: 30$ & 100 & 110 & 56 & 90 & & $40+$ \\
\hline $5 / 29-11: 35$ & 88 & 110 & 56 & 90 & 659 & $40+$ \\
\hline$5 \longdiv { 2 9 - 1 2 : 0 0 }$ & 90 & 120 & 56 & 90 & 61.3 & $4 \overline{0}+$ \\
\hline $5 / 30-08 \cdot 20$ & 104 & 110 & 56 & 80 & 55.4 & $40+$ \\
\hline $5 / 30-09: 25$ & 104 & 120 & & & & \\
\hline $5 / 30-11: 20$ & 105 & 120 & 51 & 80 & 56.5 & $40+$ \\
\hline $5 / 30-12: 15$ & 105 & 120 & 50 & 80 & 65.6 & $40+$ \\
\hline $5 / 30-13: 15$ & 105 & 120 & 50 & 80 & 59.4 & $40+$ \\
\hline $6 / 3-10 \cdot 00$ & 102 & 120 & & 85 & 556 & $40+$ \\
\hline $6 / 3-10: 15$ & 102 & 105 & & 75 & 56.3 & $40+$ \\
\hline $6 / 3-10: 45$ & 102 & 105 & & 75 & 55.8 & $40+$ \\
\hline $6 / 3-11: 30$ & 104 & 110 & & 80 & 53.7 & $40+$ \\
\hline & & & & & & \\
\hline $6 / 4-09: 40$ & 99 & 120 & 50 & 90 & 71.4 & $40 t$ \\
\hline $6 / 4-10: 00$ & 100 & 110 & 50 & $\overline{80}$ & 59.5 & $40+$ \\
\hline $6 / 4-10: 15$ & $10 \overline{0}$ & 110 & 50 & 80 & 61.3 & $40+$ \\
\hline $6 / 4-11: 15$ & 100 & 105 & 49 & 80 & 62.4 & $40+$ \\
\hline & & & & & & \\
\hline $6 / 6-11: 55$ & 102 & 120 & 50 & 80 & 73.5 & $40+$ \\
\hline $6 / 6-12: 55$ & 102 & 115 & 49 & 75 & 67.8 & $40+$ \\
\hline $6 / 10-09: 30$ & 104 & 110 & 50 & 70 & 685 & $40+$ \\
\hline $6 / 10-09: 45$ & 104 & 110 & 50 & 80 & 62.6 & $40+$ \\
\hline $6 / 10-10: 35$ & 120 & 120 & 50 & 85 & 62 & $40+$ \\
\hline $6 / 10-11: 30$ & 120 & 120 & 50 & 85 & 63.5 & $40+$ \\
\hline $6 / 10-12: 30$ & 120 & 120 & 50 & 80 & 57.4 & $40+$ \\
\hline $6 / 10-13: 30$ & 110 & 110 & 50 & 80 & 60.9 & $40+$ \\
\hline $6 / 11-00 \cdot 15$ & 103 & 110 & 50 & 75 & 731 & $\Delta 0+$ \\
\hline $6 / 11-10: 00$ & 104 & 110 & 50 & 75 & 69 & $40+$ \\
\hline & & & & & & \\
\hline $6 / 13-12: 15$ & 106 & 120 & 50 & 80 & 64.9 & $40+$ \\
\hline $6 / 13-12: 40$ & 106 & 110 & 49 & 75 & 63.8 & $40+$ \\
\hline $6 / 13-13: 15$ & 108 & 110 & 49 & 80 & 70 & $40+$ \\
\hline $6 / 16-10: 45$ & 94 & 110 & 48 & 65 & 75.8 & $40+$ \\
\hline $6 / 16-11: 20$ & 94 & 110 & 47 & 65 & 78.4 & $40+$ \\
\hline $6 / 16-13: 20$ & 108 & 110 & 47 & 70 & 71.5 & $40+$ \\
\hline & & & & & & \\
\hline $6 / 18-11: 40$ & 105 & 110 & 48 & 70 & 80 & $40+$ \\
\hline $6 / 18-12: 00$ & 107 & 110 & 48 & 70 & 70.4 & $40+$ \\
\hline & & & & & & \\
\hline $6 / 19-11: 45$ & 102 & 110 & 48 & 70 & 79.5 & $40+$ \\
\hline $6 / 19-12: 00$ & 104 & 110 & 49 & 70 & 71 & $40+$ \\
\hline
\end{tabular}




\begin{tabular}{|c|c|c|c|c|c|c|}
\hline DATE-TIME & TEMP & PUMP psi & \begin{tabular}{|c|} 
PRE \\
FILTER psi
\end{tabular} & $\begin{array}{c}\text { CONC } \\
\text { PRESSURE }\end{array}$ & $\begin{array}{c}\text { CONC } \\
\text { FLOW gpm }\end{array}$ & $\begin{array}{l}\text { PERM } \\
\text { FLOW }\end{array}$ \\
\hline $6 / 20-08: 00$ & 98 & 110 & 48 & 65 & 82.4 & $40+$ \\
\hline $6 / 20-08: 15$ & 100 & 110 & 48 & 70 & 68.8 & $40+$ \\
\hline $6 / 20-11: 15$ & 100 & 110 & 48 & 70 & 72 & $40+$ \\
\hline $6 / 20-12: 15$ & 102 & 110 & 48 & 70 & 74.6 & $40+$ \\
\hline $6 / 25-07: 40$ & 104 & 110 & 48 & 70 & 84.4 & $40+$ \\
\hline $6 / 25-08: 05$ & 105 & 110 & 48 & 70 & 72.3 & $40+$ \\
\hline $6 / 25-11: 30$ & 105 & 110 & 48 & 70 & 75.3 & $40+$ \\
\hline $6 / 26-07: 30$ & 103 & 110 & 48 & 70 & 67.6 & $40+$ \\
\hline $6 / 26-08: 15$ & 104 & 110 & 48 & 70 & 70.3 & $40+$ \\
\hline $6 / 26-12: 15$ & 108 & 110 & 48 & 70 & 74 & $40+$ \\
\hline $6 / 30-08: 40$ & 87 & 110 & 47 & 60 & 95.7 & $40+$ \\
\hline $6 / 30-08: 50$ & 88 & 110 & 48 & 65 & 86.2 & $40+$ \\
\hline $6 / 30-09: 50$ & 90 & 110 & 48 & 70 & 82.6 & $40+$ \\
\hline $6 / 30-10 ; 15$ & 91 & 110 & 48 & 70 & 78.4 & $40+$ \\
\hline $6 / 30-12: 30$ & 96 & 110 & 48 & 70 & 84.6 & $40+$ \\
\hline $6 / 3012: 45$ & 99 & 110 & 48 & 70 & 81.1 & $40+$ \\
\hline $7 / 1-08: 00$ & 95 & 110 & 47 & 70 & 832 & $40+$ \\
\hline $7 / 1-08: 15$ & 97 & 110 & 47 & 70 & 82.3 & $40+$ \\
\hline $7 / 1-08: 43$ & 99 & 110 & 48 & 70 & 78.9 & $40+$ \\
\hline $7 / 1-10: 45$ & 102 & 110 & 48 & 70 & 80.3 & $40+$ \\
\hline $7 / 1-13: 30$ & 104 & 110 & 48 & 70 & 82 & $40+$ \\
\hline $7 / 2-09: 37$ & 99 & 220 & 50 & 180 & 84.4 & $40+$ \\
\hline $7 / 2-10: 15$ & 102 & 215 & 50 & 180 & 87.5 & $40+$ \\
\hline $7 / 2-10: 45$ & 105 & 220 & 50 & 180 & 85.4 & $40+$ \\
\hline $7 / 2-12: 15$ & 106 & 230 & 50 & 190 & 88.3 & $40+$ \\
\hline $7 / 2-12: 45$ & 108 & 225 & 50 & 190 & 84.7 & $40+$ \\
\hline & & & & & & \\
\hline $7 / 9-09: 45$ & 104 & 220 & 51 & 200 & 54.7 & $40+$ \\
\hline $7 / 9-10: 05$ & 104 & 230 & 51 & 200 & 54.5 & $40+$ \\
\hline $7 / 9-11: 45$ & 107 & 230 & 50 & 200 & 63.7 & $40+$ \\
\hline $7 / 9-12: 45$ & 110 & 230 & 50 & 200 & 69.8 & $40+$ \\
\hline $7 / 10-09: 45$ & 102 & 230 & 50 & 200 & 68.5 & $40+$ \\
\hline $7 / 10-10: 05$ & 104 & 230 & 50 & 200 & 68.4 & $40+$ \\
\hline $7 / 11-07 \cdot 30$ & 100 & 230 & 50 & 200 & 715 & $40 t$ \\
\hline $7 / 11-07: 45$ & 101 & 230 & 50 & 200 & 75.5 & $40+$ \\
\hline $7 / 11-08: 15$ & 104 & 230 & 50 & 200 & 74.2 & $40+$ \\
\hline $7 / 11-10: 30$ & 104 & 230 & 50 & 200 & 81.3 & $40+$ \\
\hline $7 / 11-10: 50$ & 106 & 230 & 50 & 200 & 69 & $40+$ \\
\hline $7 / 11-11: 45$ & 107 & 230 & 50 & 200 & 70.6 & $40+$ \\
\hline $7 / 11-12: 15$ & 110 & 230 & 50 & 200 & 81.8 & $40+$ \\
\hline & & & & & & \\
\hline
\end{tabular}


OPERATIONAL DATA

\begin{tabular}{|c|c|c|c|c|c|c|}
\hline DATE-TIME & TEMP & PUMP psi & PRE & CONC & CONC & PERM \\
\hline & & & FILTER ps & PRESSURE & FLOW gpm & FLOW \\
\hline $7 / 15-09: 45$ & 103 & 230 & 50 & 200 & 59 & $40+$ \\
\hline $7 / 15-10: 00$ & 106 & 230 & 50 & 200 & 59.6 & $40+$ \\
\hline $7 / 15-11: 30$ & 107 & 230 & 50 & 200 & 61.9 & $40+$ \\
\hline $7 / 15-11: 45$ & 112 & 230 & 50 & 200 & 57 & $40+$ \\
\hline $7 / 15-13: 30$ & 110 & 230 & 50 & 200 & 61.4 & $40+$ \\
\hline $7 / 16-09: 35$ & 107 & 230 & 50 & 200 & 616 & $\Delta 0+$ \\
\hline $7 / 16-10: 10$ & 110 & 230 & 50 & 200 & 66.9 & $40+$ \\
\hline
\end{tabular}

Page 3 